\title{
Lower bounds for the first eigenvalue of the magnetic Laplacian
}

\author{
Bruno Colbois and Alessandro Savo
}

July 2, 2018

\begin{abstract}
We consider a Riemannian cylinder $\Omega$ endowed with a closed potential 1-form $A$ and study the magnetic Laplacian $\Delta_{A}$ with magnetic Neumann boundary conditions associated with those data. We establish a sharp lower bound for the first eigenvalue and show that the equality characterizes the situation where the metric is a product. We then look at the case of a planar domain bounded by two closed curves and obtain an explicit lower bound in terms of the geometry of the domain. We finally discuss sharpness of this last estimate.
\end{abstract}

2000 Mathematics Subject Classification. 58J50, 35P15.

Key words and phrases. Magnetic Laplacian, Eigenvalues, Upper and lower bounds, Zero magnetic field

\section{Introduction}

Let $(\Omega, g)$ be a compact Riemannian manifold with boundary. Consider the trivial complex line bundle $\Omega \times \mathbf{C}$ over $\Omega$; its space of sections can be identified with $C^{\infty}(\Omega, \mathbf{C})$, the space of smooth complex valued functions on $\Omega$. Given a smooth real 1-form $A$ on $\Omega$ we define a connection $\nabla^{A}$ on $C^{\infty}(\Omega, \mathbf{C})$ as follows:

$$
\nabla_{X}^{A} u=\nabla_{X} u-i A(X) u
$$

for all vector fields $X$ on $\Omega$ and for all $u \in C^{\infty}(\Omega, \mathbf{C})$; here $\nabla$ is the Levi-Civita connection assocated to the metric $g$ of $\Omega$. The operator

$$
\Delta_{A}=\left(\nabla^{A}\right)^{\star} \nabla^{A}
$$

is called the magnetic Laplacian associated to the magnetic potential $A$, and the smooth two form

$$
B=d A
$$

is the associated magnetic field. We will consider Neumann magnetic conditions, that is:

$$
\nabla_{N}^{A} u=0 \quad \text { on } \quad \partial \Omega
$$


where $N$ denotes the inner unit normal. Then, it is well-known that $\Delta_{A}$ is self-adjoint, and admits a discrete spectrum

$$
0 \leq \lambda_{1}\left(\Delta_{A}\right) \leq \lambda_{2}\left(\Delta_{A}\right) \leq \ldots \rightarrow \infty
$$

The above is a particular case of a more general situation, where $E \rightarrow M$ is a complex line bundle with a hermitian connection $\nabla^{E}$, and where the magnetic Laplacian is defined as $\Delta_{E}=\left(\nabla^{E}\right)^{\star} \nabla^{E}$.

The spectrum of the magnetic Laplacian is very much studied in analysis (see for example [3] and the references therein) and in relation with physics. For Dirichlet boundary conditions, lower estimates of its fundamental tone have been worked out, in particular, when $\Omega$ is a planar domain and $B$ is the constant magnetic field; that is, when the function $\star B$ is constant on $\Omega$ (see for example a Faber-Krahn type inequality in [8] and the recent [11] and the references therein, also for Neumann boundary condition). The case when the potential $A$ is a closed 1-form is particularly interesting from the physical point of view (Aharonov-Bohm effect), and also from the geometric point of view. For Dirichlet boundary conditions, there is a serie of papers for domains with a pole, when the pole approaches the boundary (see [1, 12] and the references therein). Last but not least, there is a Aharonov-Bohm approach to the question of nodal and minimal partitions, see chapter 8 of [4].

For Neumann boundary conditions, we refer in particular to the paper [9], where the authors study the multiplicity and the nodal sets corresponding to the ground state $\lambda_{1}$ for non-simply connected planar domains with harmonic potential (see the discussion below).

Let us also mention the recent article [10] (chapter 7) where the authors establish a Cheeger type inequality for $\lambda_{1}$; that is, they find a lower bound for $\lambda_{1}\left(\Delta_{A}\right)$ in terms of the geometry of $\Omega$ and the potential $A$. In the preprint [7], the authors approach the problem via the Bochner method.

Finally, in a more general context (see [2]) the authors establish a lower bound for $\lambda_{1}\left(\Delta_{A}\right)$ in terms of the holonomy of the vector bundle on which $\Delta_{A}$ acts. In both cases, implicitly, the flux of the potential $A$ plays a crucial role.

- From now on we will denote by $\lambda_{1}(\Omega, A)$ the first eigenvalue of $\Delta_{A}$ on $(\Omega, g)$.

\subsection{Main lower bound}

Our lower bound is partly inspired by the results in [9] for plane domains. First, recall that if $c$ is a closed parametrized curve (a loop), the quantity:

$$
\Phi_{c}^{A}=\frac{1}{2 \pi} \oint_{c} A
$$


is called the flux of $A$ across $c$. (We assume that $c$ is travelled once, and we will not specify the orientation of the loop, so that the flux will only be defined up to sign: this will not affect any of the statements, definitions or results which we will prove in this paper). Let then $\Omega$ be a fixed plane domain with one hole, and let $\Phi^{A}$ be the flux of the harmonic potential $A$ across the inner boundary curve. In Theorem 1.1 of [9] it is first remarked that $\lambda_{1}(\Omega, A)$ is positive if and only if $\Phi^{A}$ is not an integer (but see the precise statement in Section 2.1 below). Then, it is shown that $\lambda_{1}(\Omega, A)$ is maximal precisely when $\Phi^{A}$ is congruent to $\frac{1}{2}$ modulo integers. The proof relies on a delicate argument involving the nodal line of a first eigenfunction; in particular, the conclusion does not follow from a specific comparison argument, or from an explicit lower bound.

In this paper we give a geometric lower bound of $\lambda_{1}(\Omega, A)$ when $\Omega$ is, more generally, a Riemannian cylinder, that is, a domain $(\Omega, g)$ diffeomorphic to $[0,1] \times \mathbf{S}^{1}$ endowed with a Riemannian metric $g$, and when $A$ is a closed potential 1-form : hence, the magnetic field $B$ associated to $A$ is equal to 0 . The lower bound will depend on the geometry of $\Omega$ and, in an explicit way, on the flux of the potential $A$.

Let us write $\partial \Omega=\Sigma_{1} \cup \Sigma_{2}$ where

$$
\Sigma_{1}=\{0\} \times \mathbf{S}^{1}, \quad \Sigma_{2}=\{1\} \times \mathbf{S}^{1} .
$$

We will need to foliate the cylinder by the (regular) level curves of a smooth function $\psi$ and then we introduce the following family of functions.

$$
\begin{aligned}
\mathcal{F}_{\Omega}=\{\psi: \Omega \rightarrow \mathbf{R}: & \psi \text { is constant on each boundary component } \\
& \text { and has no critical points inside } \Omega\}
\end{aligned}
$$

As $\Omega$ is a cylinder, we see that $\mathcal{F}_{\Omega}$ is not empty. If $\psi \in \mathcal{F}_{\Omega}$, we set:

$$
K=K_{\Omega, \psi}=\frac{\sup _{\Omega}|\nabla \psi|}{\inf _{\Omega}|\nabla \psi|} .
$$

It is clear that, in the definition of the constant $K$, we can assume that the range of $\psi$ is the interval $[0,1]$, and that $\psi=0$ on $\Sigma_{1}$ and $\psi=1$ on $\Sigma_{2}$. Note that the level curves of the function $\psi$ are all smooth, closed and connected; moreover they are all homotopic to each other so that the flux of a closed 1-form $A$ across any of them is the same, and will be denoted by $\Phi^{A}$.

We say, briefly, that $\Omega$ is $K$-foliated by the level curves of $\psi$. We also denote by $d\left(\Phi^{A}, \mathbf{Z}\right)$ the minimal distance between $\Phi^{A}$ and the set of integer $\mathbf{Z}$ :

$$
d\left(\Phi^{A}, \mathbf{Z}\right)^{2}=\min \left\{\left(\Phi^{A}-k\right)^{2}: k \in \mathbf{Z}\right\} .
$$

Finally, we say that $\Omega$ is a Riemannian product if it is isometric to $[0, a] \times \mathbf{S}^{1}(R)$ for suitable positive constants $a, R$. 


\section{Theorem 1.}

a) Let $(\Omega, g)$ be a Riemannian cylinder, and let $A$ be a closed 1-form on $\Omega$. Assume that $\Omega$ is $K$-foliated by the level curves of the smooth function $\psi \in \mathcal{F}_{\Omega}$. Then:

$$
\lambda_{1}(\Omega, A) \geq \frac{4 \pi^{2}}{K L^{2}} \cdot d\left(\Phi^{A}, \mathbf{Z}\right)^{2}
$$

where $L$ is the maximum length of a level curve of $\psi$ and $\Phi^{A}$ is the flux of $A$ across any of the boundary components of $\Omega$.

b) Equality holds if and only if the cylinder $\Omega$ is a Riemannian product.

- It is clear that we can also state the lower bound as follows:

$$
\lambda_{1}(\Omega, A) \geq \frac{4 \pi^{2}}{\tilde{K}_{\Omega}} \cdot d\left(\Phi^{A}, \mathbf{Z}\right)^{2},
$$

where $\tilde{K}_{\Omega}$ is an invariant depending only $\Omega$ :

$$
\tilde{K}_{\Omega}=\inf _{\psi \in \mathcal{F}_{\Omega}} K_{\Omega, \psi} L_{\psi}^{2} \quad \text { and } \quad L_{\psi}=\sup _{r \in \operatorname{range}(\psi)}\left|\psi^{-1}(r)\right| .
$$

It is is not always easy to estimate $K$. In Section 2.4 we will show how to estimate $K$ in terms of the metric tensor. Note that $K \geq 1$; we will see that in many interesting situations (for example, for revolution cylinders, or for smooth embedded tubes around a closed curve) one has in fact $K=1$.

\subsection{Doubly connected planar domains}

We now estimate the constant $K$ above when $\Omega$ is an annular region in the plane, bounded by the inner curve $\Sigma_{1}$ and the outer curve $\Sigma_{2}$.

- We assume that the inner curve $\Sigma_{1}$ is convex.

From each point $x \in \Sigma_{1}$, consider the ray $\gamma_{x}(t)=x+t N_{x}$, where $N_{x}$ is the exterior normal to $\Sigma_{1}$ at $x$ and $t \geq 0$. Let $Q(x)$ be the first intersection of $\gamma_{x}(t)$ with $\Sigma_{2}$, and let

$$
r(x)=d(x, Q(x)) .
$$

We say that $\Omega$ is starlike with respect to $\Sigma_{1}$ if the map $x \rightarrow Q(x)$ is a bijection between $\Sigma_{1}$ and $\Sigma_{2}$; equivalently, if given any point $y \in \Sigma_{2}$, the geodesic segment which minimizes distance from $y$ to $\Sigma_{1}$ is entirely contained in $\Omega$.

For $x \in \Sigma_{1}$, we denote by $\theta_{x}$ the angle between $\gamma_{x}^{\prime}$ and the outer normal to $\Sigma_{2}$ at the point $Q(x)$, and we let

$$
m \doteq \min _{x \in \Sigma_{1}} \cos \theta_{x}
$$


Note that as $\Omega$ is starlike w.r.t. $\Sigma_{1}$, one has $\theta_{x} \in\left[0, \frac{\pi}{2}\right]$ and then $m \geq 0$.

- To have a positive lower bound, we will assume that $m>0$ (that is, $\Omega$ is strictly starlike w.r.t. $\left.\Sigma_{1}\right)$.

We also define

$$
\left\{\begin{array}{l}
\beta=\min \left\{r(x): x \in \Sigma_{1}\right\} \\
B=\max \left\{r(x): x \in \Sigma_{1}\right\}
\end{array}\right.
$$

We then have the following result.

Theorem 2. Let $\Omega$ be an annulus in $\mathbf{R}^{2}$, which is strictly-starlike with respect to its inner (convex) boundary component $\Sigma_{1}$. Assume that $A$ is a closed potential having flux $\Phi^{A}$ around $\Sigma_{1}$. Then:

$$
\lambda_{1}(\Omega, A) \geq \frac{4 \pi^{2}}{L^{2}} \frac{\beta m}{B} d\left(\Phi^{A}, \mathbf{Z}\right)^{2}
$$

where $\beta$ and $B$ are as in (16), and $L$ is the length of the outer boundary component. If $\Sigma_{2}$ is also convex, then $m \geq \beta / B$ and the lower bound takes the form:

$$
\lambda_{1}(\Omega, A) \geq \frac{4 \pi^{2}}{L^{2}} \frac{\beta^{2}}{B^{2}} d\left(\Phi^{A}, \mathbf{Z}\right)^{2} .
$$

In section 4 , we will explain why we need to control $\frac{\beta}{B}, L$, and why we need to impose the starlike condition. If $\beta=B$ and $\Sigma_{2}$ is the circle of length $L$ we get the estimate

$$
\lambda_{1}(\Omega, A) \geq \frac{4 \pi^{2}}{L^{2}} d\left(\Phi^{A}, \mathbf{Z}\right)^{2}
$$

which is the first eigenvalue of the magnetic Laplacian on the circle with potential $A$ (see section 5.1). If $\Sigma_{2}$ and $\Sigma_{1}$ are two concentric circles of respective lengths $L$ and $L_{\epsilon} \rightarrow L$, the domain is a thin annulus with $\lambda_{1} \rightarrow \frac{4 \pi^{2}}{L^{2}} d\left(\Phi^{A}, \mathbf{Z}\right)^{2}$ which shows that our estimate is sharp.

Our aim is to use these estimates on cylinders as a basis stone in order to study the same type of questions on compact surfaces of higher genus.

\section{Proof of the main theorem}

\subsection{Preliminary facts and notation}

First, we recall the variational definition of the spectrum. Let $\Omega$ be a compact manifold with boundary and $\Delta_{A}$ the magnetic Laplacian with Neumann boundary conditions. One 
verifies that

$$
\int_{\Omega}\left(\Delta_{A} u\right) \bar{u}=\int_{\Omega}\left|\nabla^{A} u\right|^{2}
$$

and the associated quadratic form is then

$$
Q_{A}(u)=\int_{\Omega}\left|\nabla^{A} u\right|^{2}
$$

The usual variational characterization gives:

$$
\lambda_{1}(\Omega, A)=\min \left\{\frac{Q_{A}(u)}{\|u\|^{2}}: u \in C^{1}(\Omega, \mathbb{C}) /\{0\}\right\}
$$

The following proposition (which is well-known) expresses the gauge invariance of the spectrum of the magnetic Laplacian.

Proposition 3. a) The spectrum of $\Delta_{A}$ is equal to the spectrum of $\Delta_{A+d \phi}$ for all smooth real valued functions $\phi$; in particular, when $A$ is exact, the spectrum of $\Delta_{A}$ reduces to that of the classical Laplace-Beltrami operator acting on functions (with Neumann boundary conditions if $\partial \Omega$ is not empty).

b) If $A$ is a closed 1-form, then $A$ is gauge equivalent to a unique (harmonic) 1-form $\tilde{A}$ satisfying

$$
\left\{\begin{array}{l}
d \tilde{A}=\delta \tilde{A}=0 \quad \text { on } \quad \Omega \\
\tilde{A}(N)=0 \quad \text { on } \quad \partial \Omega
\end{array}\right.
$$

The form $\tilde{A}$ is often called the Coulomb gauge of $A$. Note that $\tilde{A}$ is the harmonic representative of $A$ for the absolute boundary conditions.

Proof. a) This comes from the fact that $\Delta_{A} e^{-i \phi}=e^{-i \phi} \Delta_{A+d \phi}$ hence $\Delta_{A}$ and $\Delta_{A+d \phi}$ are unitarily equivalent.

b) Consider a solution $\phi$ of the problem:

$$
\left\{\begin{array}{l}
\Delta \phi=\delta A \quad \text { on } \quad \Omega, \\
\frac{\partial \phi}{\partial N}=A(N) \quad \text { on } \quad \partial \Omega .
\end{array}\right.
$$

Then one checks that $\tilde{A}=A-d \phi$ is a Coulomb gauge of $A$. As $\phi$ is unique up to an additive constant, $d \phi$, hence $\tilde{A}$, is unique.

We now focus on the first eigenvalue. Clearly, if $A=0$, then $\lambda_{1}(\Omega, A)=0$ simply because $\Delta_{A}$ reduces to the usual Laplacian, which has first eigenvalue equal to zero and 
first eigenspace spanned by the constant functions. If $A$ is exact, then $\Delta_{A}$ is unitarily equivalent to $\Delta$, hence, again, $\lambda_{1}(\Omega, A)=0$. In fact one checks easily from the definition of the connection that, if $A=d \phi$ for some real-valued function $\phi$ then $\nabla^{A} e^{i \phi}=0$, which means that $u=e^{i \phi}$ is $\nabla^{A}$-parallel hence $\Delta_{A}$-harmonic. On the other hand, if the magnetic field $B=d A$ is non-zero then $\lambda_{1}(\Omega, A)>0$.

It then remains to examine the case when $A$ is closed but not exact. The situation was clarified in [13] for closed manifolds and in [9] for Neumann boundary conditions.

Theorem 4. The following statements are equivalent:

a) $\lambda_{1}(\Omega, A)=0$;

b) $d A=0$ and $\Phi_{c}^{A} \in \mathbf{Z}$ for any closed curve $c$ in $\Omega$.

Thus, the first eigenvalue vanishes if and only if $A$ is a closed form whose flux around every closed curve is an integer; equivalently, if $A$ has non-integral flux around at least one closed loop, then $\lambda_{1}(\Omega, A)>0$.

\subsection{Proof of the lower bound}

From now on we assume that $\Omega$ is a Riemannian cylinder. Fix a first eigenfunction $u$ associated to $\lambda_{1}(\Omega, A)$ and fix a level curve

$$
\Sigma_{r}=\{\psi=r\}, \quad \text { where } r \in[0,1] .
$$

As $\psi$ has no critical points, $\Sigma_{r}$ is isometric to $\mathbf{S}^{1}\left(\frac{L_{r}}{2 \pi}\right)$, where $L_{r}$ is the length of $\Sigma_{r}$. The restriction of $A$ to $\Sigma_{r}$ is a closed 1 -form denoted by $\tilde{A}$; we use the restriction of $u$ to $\Sigma_{r}$ as a test-function for the first eigenvalue $\lambda_{1}\left(\Sigma_{r}, \tilde{A}\right)$ and obtain:

$$
\lambda_{1}\left(\Sigma_{r}, \tilde{A}\right) \int_{\Sigma_{r}}|u|^{2} \leq \int_{\Sigma_{r}}\left|\nabla^{\tilde{A}} u\right|^{2} .
$$

By the estimate on the eigenvalues of a circle done in Section 2.3.3 below we see :

$$
\lambda_{1}\left(\Sigma_{r}, \tilde{A}\right)=\frac{4 \pi^{2}}{L_{r}^{2}} d\left(\Phi^{\tilde{A}}, \mathbf{Z}\right)^{2},
$$

where $\Phi^{\tilde{A}}$ is the flux of $\tilde{A}$ across $\Sigma_{r}$. Now note that $\Phi^{\tilde{A}}=\Phi^{A}$, because $\tilde{A}$ is the restriction of $A$ to $\Sigma_{r}$; moreover $L_{r} \leq L$ by the definition of $L$. Therefore:

$$
\lambda_{1}\left(\Sigma_{r}, \tilde{A}\right) \geq \frac{4 \pi^{2}}{L^{2}} d\left(\Phi^{A}, \mathbf{Z}\right)^{2}
$$


for all $r$. Let $X$ be a unit vector tangent to $\Sigma_{r}$. Then:

$$
\begin{aligned}
\nabla_{X}^{\tilde{A}} u & =\nabla_{X} u-i \tilde{A}(X) u \\
& =\nabla_{X} u-i A(X) u \\
& =\nabla_{X}^{A} u
\end{aligned}
$$

The consequence is that:

$$
\left|\nabla^{\tilde{A}} u\right|^{2}=\left|\nabla_{X}^{\tilde{A}} u\right|^{2}=\left|\nabla_{X}^{A} u\right|^{2} \leq\left|\nabla^{A} u\right|^{2} .
$$

- Note that equality holds in (9) iff $\nabla_{N}^{A} u=0$ where $N$ is a unit vector normal to the level curve $\Sigma_{r}$ (we could take $N=\nabla \psi /|\nabla \psi|$ ).

For any fixed level curve $\Sigma_{r}=\{\psi=r\}$ we then have, taking into account (7), (8) and (9):

$$
\frac{4 \pi^{2}}{L^{2}} d\left(\Phi^{A}, \mathbf{Z}\right)^{2} \int_{\psi=r}|u|^{2} \leq \int_{\psi=r}\left|\nabla^{A} u\right|^{2}
$$

Assume that $B_{1} \leq|\nabla \psi| \leq B_{2}$ for positive constants $B_{1}, B_{2}$. Then the above inequality implies:

$$
\frac{4 \pi^{2}}{L^{2}} d\left(\Phi^{A}, \mathbf{Z}\right)^{2} \cdot B_{1} \int_{\psi=r} \frac{|u|^{2}}{|\nabla \psi|} \leq B_{2} \int_{\psi=r} \frac{\left|\nabla^{A} u\right|^{2}}{|\nabla \psi|} .
$$

We now integrate both sides from $r=0$ to $r=1$ and use the coarea formula. Conclude that

$$
\frac{4 \pi^{2}}{L^{2}} d\left(\Phi^{A}, \mathbf{Z}\right)^{2} \cdot B_{1} \int_{\Omega}|u|^{2} \leq B_{2} \int_{\Omega}\left|\nabla^{A} u\right|^{2} .
$$

As $u$ is a first eigenfunction, one has:

$$
\int_{\Omega}\left|\nabla^{A} u\right|^{2}=\lambda_{1}(\Omega, A) \int_{\Omega}|u|^{2}
$$

Recalling that $K=\frac{B_{2}}{B_{1}}$ we finally obtain the estimate (4).

\subsection{Proof of the equality case}

If the cylinder $\Omega$ is a Riemannian product then it is obvious that we can take $K=1$ and then we have equality by Proposition 8 below. Now assume that we do have equality: we have to show that $\Omega$ is a Riemannian product. Going back to the proof, we must have the following facts.

F1. All level curves of $\psi$ have the same length $L$. 
F2. $|\nabla \psi|$ must be constant and, by renormalization, we can assume that it is everywhere equal to 1 . Then, $\psi: \Omega \rightarrow[0, a]$ for some $a>0$ and we set

$$
N \doteq \nabla \psi
$$

F3. The eigenfunction $u$ on $\Omega$ restricts to an eigenfunction of the magnetic Laplacian of each level set $\Sigma_{r}=\{\psi=r\}$, with potential given by the restriction of $A$ to $\Sigma_{r}$.

F4. One has $\nabla_{N}^{A} u=0$ identically on $\Omega$.

\subsubsection{First step: description of the metric}

Lemma 5. $\Omega$ is isometric to the product $[0, a] \times \mathbf{S}^{1}\left(\frac{L}{2 \pi}\right)$ with metric

$$
g=\left(\begin{array}{cc}
1 & 0 \\
0 & \theta^{2}(r, t)
\end{array}\right), \quad(r, t) \in[0, a] \times[0, L]
$$

where $\theta(r, t)$ is positive and periodic of period $L$ in the variable $t$. Moreover $\theta(0, t)=1$ for all $t$.

Proof. We first show that the integral curves of $N$ are geodesics; for this it is enough to show that $\nabla_{N} N=0$ on $\Omega$. Let $e_{1}(x)$ be a vector tangent to the level curve of $\psi$ passing through $x$. Then, we obtain a smooth vector field $e_{1}$ which, together with $N$, forms a global orthonormal frame. Now

$$
\left\langle\nabla_{N} N, N\right\rangle=\frac{1}{2} N \cdot\langle N, N\rangle=0
$$

On the other hand, as the Hessian is a symmetric tensor:

$$
\left\langle\nabla_{N} N, e_{1}\right\rangle=\nabla^{2} \psi\left(N, e_{1}\right)=\nabla^{2} \psi\left(e_{1}, N\right)=\left\langle\nabla_{e_{1}} N, N\right\rangle=\frac{1}{2} e_{1} \cdot\langle N, N\rangle=0 .
$$

Hence $\nabla_{N} N=0$ as asserted. As each integral curve of $N=\nabla \psi$ is a geodesic meeting $\Sigma_{1}$ orthogonally, we see that $\psi$ is actually the distance function to $\Sigma_{1}$. We introduce coordinates on $\Omega$ as follows. For a fixed point $p \in \Omega$ consider the unique integral curve $\gamma$ of $N$ passing through $p$ and let $x \in \Sigma_{1}$ be the intersection of $\gamma$ with $\Sigma_{1}$ (note that $x$ is the foot of the unique geodesic which minimizes the distance from $p$ to $\Sigma_{1}$ ). Let $r$ be the distance of $p$ to $\Sigma_{1}$. We then have a map $\Omega \rightarrow[0, a] \times \Sigma_{1}$ which sends $p$ to $(r, x)$. Its inverse is the map $F:[0, a] \times \Sigma_{1} \rightarrow \Omega$ defined by

$$
F(r, x)=\exp _{x}(r N)
$$

Note that $F$ is a diffeomeorphism; we call the pair $(r, x)$ the normal coordinates based on $\Sigma_{1}$. We introduce the arc-length $t$ on $\Sigma_{1}$ (with origin in any assigned point of $\Sigma_{1}$ ) 
and recall that $L$ is length of $\Sigma_{1}$ (which is also the length of $\left.\Sigma_{2}\right)$ ). Let us compute the metric $g$ in normal coordinates. Since $N=\frac{\partial}{\partial r}$ one sees that $g_{11}=1$ everywhere; for any fixed $r=r_{0}$ we have that $F\left(r_{0}, \cdot\right)$ maps $\Sigma_{1}$ diffeomorphically onto the level set $\left\{\psi=r_{0}\right\}$ so that $\frac{\partial}{\partial r}$ and $\frac{\partial}{\partial t}$ will be mapped onto orthogonal vectors, and indeed $g_{12}=0$. Setting $\theta(r, t)^{2}=\left\langle\frac{\partial}{\partial t}, \frac{\partial}{\partial t}\right\rangle$ one sees that the metric takes the form 10 . Finally note that $\theta(0, t)=1$ for all $t$, because $F(0, \cdot)$ is the identity.

\subsubsection{Second step : Gauge invariance}

Lemma 6. Let $\Omega$ be any Riemannian cylinder and $A=f(r, t) d r+h(r, t) d t$ a closed 1-form on $\Omega$. Then, there exists a smooth function $\phi$ on $\Omega$ such that

$$
A+d \phi=H(t) d t
$$

for a smooth function $H(t)$ depending only on $t$. Hence, by gauge invariance, we can assume from the start that $A=H(t) d t$.

Proof. Consider the function $\phi(r, t)=-\int_{0}^{r} f(x, t) d x$. Then:

$$
A+d \phi=\tilde{h}(r, t) d t
$$

for some smooth function $\tilde{h}(r, t)$. As $A$ is closed, also $A+d \phi$ is closed, which implies that $\frac{\partial \tilde{h}}{\partial r}=0$, that is, $\tilde{h}(t, r)$ does not depend on $r$; if we set $H(t) \doteq \tilde{h}(t, 0)$ we get the assertion.

- We point out the following consequence. If $u=u(r, t)$ is an eigenfunction, we know from $\mathbf{F} 4$ above that $\nabla_{N}^{A} u=0$, where $N=\frac{\partial}{\partial r}$. As $\nabla_{N}^{A} u=\frac{\partial u}{\partial r}-i A\left(\frac{\partial}{\partial r}\right) u$ and $A=H(t) d t$ we obtain $A\left(\frac{\partial}{\partial r}\right)=0$ hence $\frac{\partial u}{\partial r}=0$ at all points of $\Omega$. This implies that

$$
u=u(t)
$$

depends only on $t$.

\subsubsection{Third step : spectrum of circles and Riemannian products}

In this section, we give an expression for the eigenfunctions of the magnetic Laplacian on a circle with a Riemannian metric $g$ and a closed potential $A$. Of course, we know that any metric $g$ on a circle is always isometric to the canonical metric $g_{\text {can }}=d t^{2}$, 
where $t$ is arc-length. But our problem in this proof is to reconstruct the global metric of the cylinder and to show that it is a product, and we cannot suppose a priori that the restricted metric of each level set of $\psi$ is the canonical metric. The same is true for the restricted potential: we know that it is Gauge equivalent to a potential of the type $a d t$ for a scalar $a$, but we cannot suppose a priori that it is of that form.

We refer to Appendix 5.1 for the complete proof of the following fact.

Proposition 7. Let $(M, g)$ be the circle of length $L$ endowed with the metric $g=\theta(t)^{2} d t^{2}$ where $t \in[0, L]$ and $\theta(t)$ is a positive function, periodic of period $L$. Let $A=H(t) d t$. Then, the eigenvalues of the magnetic Laplacian with potential $A$ are:

$$
\lambda_{k}(M, A)=\frac{4 \pi^{2}}{L^{2}}\left(k-\Phi^{A}\right)^{2}, \quad k \in \mathbf{Z}
$$

with associated eigenfunctions

$$
u_{k}(t)=e^{i \phi(t)} e^{\frac{2 \pi i\left(k-\Phi^{A}\right)}{L} s(t)}, \quad k \in \mathbf{Z} .
$$

where $\phi(t)=\int_{0}^{t} H(\tau) d \tau$ and $s(t)=\int_{0}^{t} \theta(\tau) d \tau$.

In particular, if the metric is the canonical one, that is, $g=d t^{2}$, and the potential 1-form is harmonic, so that $A=\frac{2 \pi \Phi^{A}}{L} d t$, then the eigenfunctions are simply:

$$
u_{k}(t)=e^{\frac{2 \pi i k}{L} t}, \quad k \in \mathbf{Z} .
$$

We remark that if the flux $\Phi^{A}$ is not congruent to $1 / 2$ modulo integers, then the eigenvalues are all simple. If the flux is congruent to $1 / 2$ modulo integers, then there are two consecutive integers $k, k+1$ such that $\lambda_{k}=\lambda_{k+1}$. Consequently, the lowest eigenvalue has multiplicity two, and the first eigenspace is spanned by

$$
e^{i \phi(t)} e^{\frac{\pi i}{L} s(t)}, e^{i \phi(t)} e^{-\frac{\pi i}{L} s(t)}
$$

The following proposition is an easy consequence (for a proof, see also Appendix 5.1).

Proposition 8. Consider the Riemannian product $\Omega=[0, a] \times \mathbf{S}^{1}\left(\frac{L}{2 \pi}\right)$, and let $A$ be $a$ closed 1 -form on $\Omega$. Then, the spectrum of $\Delta_{A}$ is given by

$$
\frac{\pi^{2} h^{2}}{a^{2}}+\frac{4 \pi^{2}}{L^{2}}\left(k-\Phi^{A}\right)^{2}, \quad h, k \in \mathbf{Z}, h \geq 0 .
$$

In particular,

$$
\lambda_{1}(\Omega, A)=\frac{4 \pi^{2}}{L^{2}} d\left(\Phi^{A}, \mathbf{Z}\right)^{2} .
$$




\subsubsection{Fourth step : a calculus lemma}

In this section, we state a technical lemma which will allow us to conclude. The proof is conceptually simple, but perhaps tricky at some points; then, we decided to put it in Appendix 5.2 .

Lemma 9. Let $s:[0, a] \times[0, L] \rightarrow \mathbf{R}$ be a smooth, non-negative function such that

$$
s(0, t)=t, \quad s(r, 0)=0, \quad s(r, L)=L \quad \text { and } \quad \frac{\partial s}{\partial t}(r, t) \doteq \theta(r, t)>0 .
$$

Assume that there exist smooth functions $p(r), q(r)$ with $p(r)^{2}+q(r)^{2}>0$ such that

$$
p(r) \cos \left(\frac{\pi}{L} s(r, t)\right)+q(r) \sin \left(\frac{\pi}{L} s(r, t)\right)=F(t)
$$

where $F(t)$ depends only on $t$. Then $p$ and $q$ are constant and $\frac{\partial s}{\partial r}=0$ so that

$$
s(r, t)=t
$$

for all $(r, t)$.

\subsubsection{End of proof of the equality case}

Assume that equality holds. Then, if $u$ is an eigenfunction, we know that $u=u(t)$ by the discussion in (11) and $u$ restricts to an eigenfunction on each level circle $\Sigma_{r}$ for the potential $A=H(t) d t$ above (see Fact 3 at the beginning of Section 2.3 and the second step above).

We assume that $\Phi^{A}$ is congruent to $\frac{1}{2}$ modulo integers. This is the most difficult case; in the other cases the proof is a particular case of this, it is simpler and we omit it.

Recall that each level set $\Sigma_{r}$ is a circle of length $L$ for all $r$, with metric $g=\theta(r, t)^{2} d t$. As the flux of $A$ is congruent to $\frac{1}{2}$ modulo integers, we see that there exist complex-valued functions $w_{1}(r), w_{2}(r)$ such that

$$
u(t)=e^{i \phi(t)}\left(w_{1}(r) e^{\frac{\pi i}{L} s(r, t)}+w_{2}(r) e^{-\frac{\pi i}{L} s(r, t)}\right),
$$

which, setting $f(t)=e^{-i \phi(t)} u(t)$, we can re-write

$$
f(t)=w_{1}(r) e^{\frac{\pi i}{L} s(r, t)}+w_{2}(r) e^{-\frac{\pi i}{L} s(r, t)} .
$$

Recall that here $\phi(t)=\int_{0}^{t} H(\tau) d \tau$ and

$$
s(r, t)=\int_{0}^{t} \theta(r, \tau) d \tau .
$$


We take the real part on both sides of $(12)$ and obtain smooth real-valued functions $F(t), p(r), q(r)$ such that

$$
F(t)=p(r) \cos \left(\frac{\pi}{L} s(r, t)\right)+q(r) \sin \left(\frac{\pi}{L} s(r, t)\right) .
$$

Since $\theta(0, t)=1$ for all $t$, we see

$$
s(0, t)=t .
$$

Clearly $s(r, 0)=0$; finally, $s(r, L)=\int_{0}^{L} \theta(r, \tau) d \tau=L$, being the length of the level circle $\Sigma_{r}$. Thus, we can apply Lemma 9 and conclude that $s(r, t)=t$ for all $t$, that is,

$$
\theta(r, t)=1
$$

for all $(r, t)$ and the metric is a Riemannian product.

It might happen that $p(r)=q(r) \equiv 0$. But then the real part of $f(t)$ is zero and we can work in an analogous way with the imaginary part of $f(t)$, which cannot vanish unless $u \equiv 0$.

\subsection{General estimate of $K_{\Omega, \psi}$}

We can estimate $K_{\Omega, \psi}$ for a Riemannian cylinder $\Omega=[0, a] \times \mathbf{S}^{1}$ if we know the explicit expression of the metric in the normal coordinates $(r, t)$, where $t \in[0,2 \pi]$ is arc-length :

$$
g=\left(\begin{array}{ll}
g_{11} & g_{12} \\
g_{21} & g_{22}
\end{array}\right) .
$$

If $g^{i j}$ is the inverse matrix of $g_{i j}$, and if $\psi=\psi(r, t)$ one has:

$$
|\nabla \psi|^{2}=g^{11}\left(\frac{\partial \psi}{\partial r}\right)^{2}+2 g^{12} \frac{\partial \psi}{\partial r} \frac{\partial \psi}{\partial t}+g^{22}\left(\frac{\partial \psi}{\partial t}\right)^{2}
$$

The function $\psi(r, t)=r$ belongs to $\mathcal{F}_{\Omega}$ and one has: $|\nabla \psi|^{2}=g^{11}$, which immediately implies that we can take

$$
K_{\Omega, \psi} \leq \frac{\sup _{\Omega} g^{11}}{\inf _{\Omega} g^{11}}
$$

Note in particular that if $\Omega$ is rotationally invariant, so that the metric can be put in the form:

$$
g=\left(\begin{array}{cc}
1 & 0 \\
0 & \alpha(r)^{2}
\end{array}\right)
$$

for some function $\alpha(r)$, then $K_{\Omega, \psi}=1$. The estimate becomes

$$
\lambda_{1}(\Omega, A) \geq \frac{4 \pi^{2}}{L^{2}} \cdot d\left(\Phi^{A}, \mathbf{Z}\right)^{2},
$$

where $L$ is the maximum length of a level curve $r=$ const. 
Example 10. Yet more generally, one can fix a smooth closed curve $\gamma$ on a Riemannian surface $M$ and consider the tube of radius $R$ around $\gamma$ :

$$
\Omega=\{x \in M: d(x, \gamma) \leq R\}
$$

It is well-known that if $R$ is sufficiently small (less than the injectivity radius of the normal exponential map) then $\Omega$ is a cylinder with smooth boundary which can be foliated by the level sets of $\psi$, the distance function to $\gamma$. Clearly $|\nabla \psi|=1$ and (13) holds as well.

A concrete example where we could estimate the width $R$ is the case of a compact surface $M$ of genus $\geq 2$ and curvature $-a^{2} \leq K \leq-b^{2}, a \geq b>0$. Let $\gamma$ be a simple closed geodesic. Then, using the Gauss-Bonnet theorem, one can show that $R$ is bounded below by an explicit positive constant $R=R(\gamma, a)$, hence the $R$-neighborhood of $\gamma$ is diffeomorphic to the product $S^{1} \times(-1,1)$ (see for example [5]). If we take $\Omega$ as the Riemannian cylinder of width $R(\gamma, a)$ having one boundary component equal to $\gamma$ then we can foliate $\Omega$ with the level sets of the distance function to $\gamma$ and so $K=1$ and (13) holds, with $L$ given by the length of the other boundary component.

\section{Proof of Theorem 2: plane annuli}

Let $\Omega$ be an annulus in $\mathbf{R}^{2}$, which is starlike with respect to its inner convex boundary component $\Sigma_{1}$. Assume that $A$ is a closed potential having flux $\Phi^{A}$ around $\Sigma_{1}$. Recall that we have to show:

$$
\lambda_{1}(\Omega, A) \geq \frac{4 \pi^{2}}{L^{2}} \frac{\beta m}{B} d\left(\Phi^{A}, \mathbf{Z}\right)^{2}
$$

where $\beta, B$ and $m$ will be recalled below and $L$ is the length of the outer boundary component. If we assume that $\Sigma_{2}$ is also convex, then we show that $m \geq \beta / B$ and the lower bound takes the form:

$$
\lambda_{1}(\Omega, A) \geq \frac{4 \pi^{2}}{L^{2}} \frac{\beta^{2}}{B^{2}} d\left(\Phi^{A}, \mathbf{Z}\right)^{2} .
$$

Before giving the proof let us recall notation. For $x \in \Sigma_{1}$, the ray $\gamma_{x}$ is the geodesic segment $\gamma_{x}(t)=x+t N_{x}$, where $N_{x}$ is the exterior normal to $\Sigma_{1}$ at $x$ and $t \geq 0$. The ray $\gamma_{x}$ meets $\Sigma_{2}$ at a first point $Q(x)$, and we let $r(x)=d(x, Q(x))$. For $x \in \Sigma_{1}$, we denote by $\theta_{x}$ the angle between the ray $\gamma_{x}^{\prime}$ and the outer normal to $\Sigma_{2}$ at the point $Q(x)$, and we let

$$
m \doteq \min _{x \in \Sigma_{1}} \cos \theta_{x}
$$

We assume that $\Omega$ is strictly starlike, that is, $m>0$; in particular $Q(x)$ is unique. Recall also that:

$$
\beta=\min _{x \in \Sigma_{1}} r(x), \quad B=\max _{x \in \Sigma_{1}} r(x)
$$


We construct a suitable smooth function $\psi$ and estimate the constant $K=K_{\Omega, \psi}$ with respect to the geometry of $\Omega$. The starlike assumption implies that each point in $\Omega$ belongs to a unique ray $\gamma_{x}$. Then we can define a function $\psi: \Omega \rightarrow[0,1]$ as follows:

$$
\psi=\left\{\begin{array}{lll}
0 & \text { on } \quad \Sigma_{1} \\
1 & \text { on } & \Sigma_{2} \\
\text { linear on each ray from } \Sigma_{1} \text { to } \Sigma_{2} .
\end{array}\right.
$$

Estimates (14) and (15) now follow from Theorem 1 together with the following Proposition.

Proposition 11. a) At all points of $\Omega$ one has: $\frac{1}{B} \leq|\nabla \psi| \leq \frac{1}{\beta m}$. Therefore:

$$
K_{\Omega, \psi}=\frac{\sup _{\Omega}|\nabla \psi|}{\inf _{\Omega}|\nabla \psi|} \leq \frac{B}{\beta m} .
$$

b) One has

$$
\sup _{r \in[0,1]}\left|\psi^{-1}(r)\right|=L=\left|\Sigma_{2}\right| .
$$

c) If $\Sigma_{2}$ is also convex, then $m \geq \beta / B$ hence we can take $K=\beta^{2} / B^{2}$.

The proof of the Proposition 11 depends on the following steps.

Step 1. On the ray $\gamma_{x}$ joining $x$ to $Q(x)$, consider the point $Q_{t}(x)$ at distance $t$ from $x$, and let $\theta_{x}(t)$ be the angle between $\gamma_{x}^{\prime}$ and $\nabla \psi\left(Q_{t}(x)\right)$. Then the function

$$
h(t)=\cos \left(\theta_{x}(t)\right)
$$

is non-increasing in t. As $\theta_{x}(r(x))=\theta_{x}$ we have in particular:

$$
\cos \left(\theta_{x}(t)\right) \geq \cos \left(\theta_{x}\right) \geq m
$$

for all $t \in[0, r(x)]$ and $x \in \Sigma_{1}$.

Step 2. The function $r \rightarrow\left|\psi^{-1}(r)\right|$ is non-decreasing in $r$.

Step 3. If $\Sigma_{2}$ is also convex we have $m \geq \beta / B$.

We will prove Steps 1-3 below.

Proof of Proposition 11. a) At any point of $\Omega$, let $\nabla^{R} \psi$ denote the radial part of $\nabla \psi$, which is the gradient of the restriction of $\psi$ to the ray passing through the given point. As such restriction is a linear function, one sees that

$$
\frac{1}{B} \leq\left|\nabla^{R} \psi\right| \leq \frac{1}{\beta} .
$$


Since $|\nabla \psi| \geq\left|\nabla^{R} \psi\right|$ one gets immediately

$$
|\nabla \psi| \geq \frac{1}{B}
$$

Note that $\theta_{x}(t)$, as defined above, is precisely the angle between $\nabla \psi$ and $\nabla^{R} \psi$, so that, using Step 1,

$$
\left|\nabla^{R} \psi\right|=|\nabla \psi| \cos \theta_{x}(t) \geq m|\nabla \psi|
$$

hence:

$$
|\nabla \psi| \leq \frac{1}{m}\left|\nabla^{R} \psi\right| \leq \frac{1}{\beta m} .
$$

as asserted. It is clear that b) and c) are immediate consequences of Steps 2-3.

Proof of Step 1. We use a suitable parametrization of $\Omega$. Let $l$ be the length of $\Sigma_{1}$ and consider a parametrization $\gamma:[0, l] \rightarrow \Sigma_{1}$ by arc-length $s$ with origin at a given point in $\Sigma_{1}$. Let $N(s)$ be the outer normal vector to $\Sigma_{1}$ at the point $\gamma(s)$. Consider the set:

$$
\tilde{\Omega}=\{(t, s) \in[0, \infty) \times[0, l): t \leq \rho(s)\}
$$

where we have set $\rho(s)=r(\gamma(s))$. The starlike property implies that the map $\Phi: \tilde{\Omega} \rightarrow \Omega$ defined by

$$
\Phi(t, s)=\gamma(s)+t N(s)
$$

is a diffeomorphism. Let us compute the Euclidean metric tensor in the coordinates $(t, s)$. Write $\gamma^{\prime}(s)=T(s)$ for the unit tangent vector to $\gamma$ and observe that $N^{\prime}(s)=k(s) T(s)$, where $k(s)$ is the curvature of $\Sigma_{1}$ which is everywhere non-negative because $\Sigma_{1}$ is convex. Then:

$$
\left\{\begin{array}{l}
d \Phi\left(\frac{\partial}{\partial t}\right)=N(s) \\
d \Phi\left(\frac{\partial}{\partial s}\right)=(1+t k(s)) T(s)
\end{array}\right.
$$

If we set $\Theta(t, s)=1+t k(s)$ the metric tensor is:

$$
g=\left(\begin{array}{cc}
1 & 0 \\
0 & \Theta^{2}
\end{array}\right)
$$

and an orthonormal basis is then $\left(e_{1}, e_{2}\right)$, where

$$
e_{1}=\frac{\partial}{\partial t}, \quad e_{2}=\frac{1}{\Theta} \frac{\partial}{\partial s} .
$$


In these coordinates, our function $\psi$ is written:

$$
\psi(t, s)=\frac{t}{\rho(s)} .
$$

Now

$$
\left\{\begin{array}{l}
\left\langle\nabla \psi, e_{1}\right\rangle=\frac{\partial \psi}{\partial t}=\frac{1}{\rho(s)} \\
\left\langle\nabla \psi, e_{2}\right\rangle=\frac{1}{\Theta} \frac{\partial \psi}{\partial s}=-\frac{t \rho^{\prime}(s)}{\Theta(t, s) \rho(s)^{2}}
\end{array} .\right.
$$

It follows that

$$
|\nabla \psi|^{2}=\frac{1}{\rho^{2}}+\frac{t^{2} \rho^{\prime 2}}{\Theta^{2} \rho^{4}}=\frac{\Theta^{2} \rho^{2}+t^{2} \rho^{\prime 2}}{\Theta^{2} \rho^{4}} .
$$

Recall the radial gradient, which is the orthogonal projection of $\nabla \psi$ on the ray, whose direction is given by $e_{1}$. If we fix $x \in \Sigma_{1}$, we have

$$
\theta_{x}(t)=\text { angle between } \nabla \psi \text { and } e_{1}
$$

and we have to study the function

$$
h(t)=\cos \theta_{x}(t)=\frac{\left\langle\nabla \psi, e_{1}\right\rangle}{|\nabla \psi|}=\frac{1}{\rho(s)|\nabla \psi|}
$$

for a fixed $s$. From the above expression of $|\nabla \psi|$ and a suitable manipulation we see

$$
h(t)^{2}=\frac{\Theta^{2}}{\Theta^{2}+t^{2} g^{2}}
$$

where $g=\rho^{\prime}(s) / \rho(s)$. Now

$$
\frac{d}{d t} \frac{\Theta^{2}}{\Theta^{2}+t^{2} g^{2}}=\frac{2 t \Theta g^{2}}{\left(\Theta^{2}+t^{2} g^{2}\right)^{2}}\left(t \frac{\partial \Theta}{\partial t}-\Theta\right)
$$

As $\Theta(t, s)=1+t k(s)$ one sees that $t \frac{\partial \Theta}{\partial t}-\Theta=-1$ hence

$$
\frac{d}{d t} h(t)^{2}=-\frac{2 t \Theta g^{2}}{\left(\Theta^{2}+t^{2} g^{2}\right)^{2}} \leq 0
$$

Hence $h(t)^{2}$ is non-increasing and, as $h(t)$ is positive, it is itself non-increasing.

Proof of Step 2. In the coordinates $(t, s)$ the curve $\psi^{-1}(r)$ is parametrized by $\alpha:[0, l] \rightarrow$ $\tilde{\Omega}$ as follows:

$$
\alpha(u)=(r \rho(u), u) \quad u \in[0, l]
$$


Then:

$$
\begin{aligned}
\left|\psi^{-1}(r)\right| & =\int_{0}^{l} \sqrt{g\left(\alpha^{\prime}(u), \alpha^{\prime}(u)\right)} d u \\
& =\int_{0}^{l} \sqrt{r^{2} \rho^{\prime}(u)^{2}+(1+r k(u) \rho(u))^{2}} d u
\end{aligned}
$$

Convexity of $\Sigma_{1}$ implies that $k(u) \geq 0$ for all $u$; differentiating under the integral sign with respect to $r$ one sees that indeed $\frac{d}{d r}\left|\psi^{-1}(r)\right| \geq 0$ for all $r \in[0,1]$.

Proof of Step 3. Let $T_{x}$ be the tangent line to $\Sigma_{2}$ at $Q(x)$ and $H(x)$ the point of $T_{x}$ closest to $x$. As $\Sigma_{2}$ is convex, $H(x)$ is not an interior point of $\Omega$, hence

$$
d(x, H(x)) \geq \beta .
$$

The triangle formed by $x, Q(x)$ and $H(x)$ is rectangle in $H(x)$, then we have:

$$
r(x) \cos \theta_{x}=d(x, H(x)) .
$$

As $r(x) \leq B$ we conclude:

$$
B \cos \theta_{x} \geq \beta
$$

which gives the assertion.

\section{Sharpness of the lower bound}

\subsection{An upper bound}

In this short paragraph, we give a simple way to get an upper bound when the potential $A$ is closed. Then, we will use this in different kinds of examples, in order to show that the assumptions of Theorem 2 are sharp. The geometric idea is the following: if we have a region $D \subset \Omega$ such that the first absolute cohomology group $H^{1}(D)$ is 0 , then we can estimate from above the spectrum of $\Delta_{A}$ in $\Omega$ in terms of the spectrum of the usual Laplacian on $D$. The reason is that the potential $A$ is 0 on $D$ up to a gauge transformation; then, on $D, \Delta_{A}$ becomes the usual Laplacian and any eigenfunction of the Laplacian on $D$ may be extended by 0 on $\Omega$ and thus used as a test function for the magnetic Laplacian on the whole of $\Omega$.

Let us give the details. Let $D$ be a closed subset of $\Omega$ such that, for some (small) $\delta>0$ one has $H^{1}\left(D^{\delta}, \mathbf{R}\right)=0$, where $D^{\delta}=\{p \in \Omega$ : $\operatorname{dist}(p, D)<\delta\}$. This happens when $D^{\delta}$ has a retraction onto $D$. We write

$$
\partial D=(\partial D \cap \partial \Omega) \cup(\partial D \cap \Omega)=\partial^{\text {ext }} D \cup \partial^{\text {int }} D
$$


and we denote by $\left(\nu_{j}(D)\right)_{j=1}^{\infty}$ the spectrum of the Laplacian acting on functions, with the Neumann boundary condition on $\partial^{\text {ext }} D$ (if non empty) and the Dirichlet boundary condition on $\partial^{\text {int }} D$.

Proposition 12. Let $\Omega$ be a compact manifold with smooth boundary and $A$ a closed potential on $\Omega$. Assume that $D \subset \Omega$ is a compact subdomain such that $H^{1}(D, \boldsymbol{R})=$ $H^{1}\left(D^{\delta}, \boldsymbol{R}\right)=0$ for some $\delta>0$. Then we have

$$
\lambda_{k}(\Omega, A) \leq \nu_{k}(D)
$$

for each $k \geq 1$.

Proof. We recall that for any function $\phi$ on $\Omega$, the operator $\Delta_{A}$ and $\Delta_{A+d \phi}$ are unitarily equivalent and have the same spectrum. As $A$ is closed and, by assumption, $H^{1}\left(D^{\delta}, \mathbf{R}\right)=$ $0, A$ is exact on $D^{\delta}$ and there exists a function $\tilde{\phi}$ on $D^{\delta}$ such that $A+d \tilde{\phi}=0$ on $D^{\delta}$. We consider the restriction of $\tilde{\phi}$ to $D$ and extend it differentiably on $\Omega$ by using a partition of unity $\left(\chi_{1}, \chi_{2}\right)$ subordinated to $\left(D^{\delta}, \Omega / D\right)$. Then, setting

$$
\phi \doteq \chi_{1} \tilde{\phi}
$$

we see that $\phi$ is a smooth function on $\Omega$ which is equal to $\tilde{\phi}$ on $D$ so that, on $D$, one has $A+d \phi=0$. We consider the new potential $\tilde{A}=A+d \phi$ and observe that $\tilde{A}=0$ on $D$.

Now consider an eigenfunction $f$ for the mixed problem on $D$ (Neumann boundary conditions on $\partial^{\text {ext }} D$ and Dirichlet boundary conditions on $\partial^{\text {int }} D$ ), and extend it by 0 on $\Omega \backslash D$. As $\tilde{A}=0$ on $D$, we see that

$$
\left|\nabla^{\tilde{A}} f\right|^{2}=|\nabla f|^{2}
$$

and we get a test function having the same Rayleigh quotient as that of $f$. Thanks to the usual min-max characterization of the spectrum, we obtain, for all $k$ :

$$
\lambda_{k}(\Omega, A)=\lambda_{k}(\Omega, \tilde{A}) \leq \nu_{k}(D) .
$$

\subsection{Sharpness}

We will use Proposition 12 to show the sharpness of the hypothesis in Theorem 2, Let us first show that we need to control the ratio $\frac{B L}{\beta}$.

Example 13. In the first situation, we give an example where the ratio $\frac{B L}{\beta} \rightarrow \infty$ and the distance $\beta$ between the two components of the boundary is uniformly bounded from below. We want to show that $\lambda_{1} \rightarrow 0$. We consider an annulus $\Omega$ composed of two concentric balls of radius 1 and $R+1$ and same center, with $R \rightarrow \infty$. We have $B=\beta=R$ and $L \rightarrow \infty$. 
From the assumptions we get the existence of a point $x \in \Omega$ such that the ball $B\left(x, \frac{R}{2}\right)$ of center $x$ and radius $\frac{R}{2}$ is contained in $\Omega$. Proposition 12 implies that $\lambda_{1}(\Omega, A)$ is bounded from above by the first eigenvalue of the Dirichlet problem for the Laplacian of the ball, which is proportional to $\frac{1}{R^{2}}$ and tends to zero because $R \rightarrow \infty$.

Example 14. Next, we construct an example to show that if the distance $\beta$ tends to 0 and $B$ and $L$ are uniformly bounded from below and from above, then again $\lambda_{1} \rightarrow 0$. We again use Proposition 12. Fix the rectangles :

$$
R_{2}=[-4,4] \times[0,4], \quad R_{1, \epsilon}=[-3,3] \times[\epsilon, 2]
$$

and consider the region $\Omega_{\epsilon}$ given by the closure of $R_{2} \backslash R_{1, \epsilon}$. Note that $\Omega_{\epsilon}$ is a planar annulus whose boundary components are convex and get closer and closer as $\epsilon \rightarrow 0$.

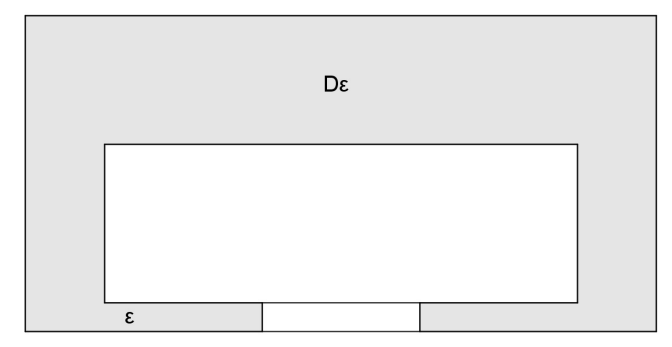

Figure 1: $\lambda_{1} \rightarrow 0$ as $\epsilon \rightarrow 0$

We show that, for any closed potential $A$ one has:

$$
\lim _{\epsilon \rightarrow 0} \lambda_{1}\left(\Omega_{\epsilon}, A\right)=0 .
$$

Consider the simply connected region $D_{\epsilon} \subset \Omega_{\epsilon}$ given by the complement of the rectangle $[-1,1] \times[0, \epsilon]$. Now $D_{\epsilon}$ has trivial 1-cohomology; by Proposition 12, to show (17) it is enough to show that

$$
\lim _{\epsilon \rightarrow 0} \nu_{1}\left(D_{\epsilon}\right)=0
$$

By the min-max principle :

$$
\nu_{1}\left(D_{\epsilon}\right)=\inf \left\{\frac{\int_{D_{\epsilon}}|\nabla f|^{2}}{\int_{D_{\epsilon}} f^{2}}: f=0 \text { on } \partial D_{\epsilon}^{\text {int }}\right\}
$$

where

$$
\partial D_{\epsilon}^{\mathrm{int}}=\left\{(x, y) \in \Omega_{\epsilon}: x= \pm 1, y \in[0, \epsilon]\right\} .
$$


Define the test-function $f: D_{\epsilon} \rightarrow \mathbf{R}$ as follows.

$$
f=\left\{\begin{array}{l}
1 \text { on the complement of }[-2,2] \times[0, \epsilon] \\
x-1 \text { on }[1,2] \times[0, \epsilon] \\
-x-1 \text { on }[-2,-1] \times[0, \epsilon]
\end{array}\right.
$$

One checks easily that, for all $\epsilon$ :

$$
\int_{D_{\epsilon}}|\nabla f|^{2}=2 \epsilon, \quad \int_{D_{\epsilon}} f^{2} \geq \text { const }>0
$$

Then (18) follows immediately by observing that the Rayleigh quotient of $f$ tends to 0 as $\epsilon \rightarrow 0$

Example 15. In the example we constructed previously the two boundary components approach each other along a common set of positive measure (precisely, a segment of total length 6). In the next example we sketch a construction showing that, in fact, this is not necessary.

So, let us fix the outside curve $\Sigma_{2}$ and choose a family of inner convex curves $\Sigma_{1}$ such that $B$ is bounded below (say, $B \geq 1$ ) and $\beta \rightarrow 0$ (no other assumption is made). Then, we want to show that $\lambda_{1}(\Omega, A) \rightarrow 0$.

Fix points $x \in \Sigma_{2}, y \in \Sigma_{1}$ such that $d(x, y)=\beta$. We take $b=2 \beta$ and introduce the balls of center $x$ and radius $b$ and $\sqrt{b}$, denoted by $B(x, b)$ and $B(x, \sqrt{b})$, respectively. Then the set $D=\Omega \backslash(B(x, b) \cap \Omega)$ is simply connected so that, by Proposition 12 ;

$$
\lambda_{1}(\Omega, A) \leq \nu_{1}(D)
$$

and it remains to show that $\nu_{1}(D) \rightarrow 0$ as $b \rightarrow 0$.

Introduce the function $F(r)$ ( $r$ being the distance to $x)$ :

$$
F(r)=\left\{\begin{array}{l}
1 \quad \text { on the complement of } B(x, \sqrt{b}) \\
0 \text { on } B(x, b) \\
\frac{-2}{\ln b}(\ln r-\ln b) \text { on } B(x, \sqrt{b})-B(x, b)
\end{array}\right.
$$

and let $f$ be the restriction of $F$ to $D$. As $f=0$ on $\partial^{\text {int }} D=\partial B(x, b) \cap \Omega$, we see that $f$ is a test function for the eigenvalue $\nu_{1}(D)$. A straightforward calculation shows that, as $b \rightarrow 0$, we have

$$
\int_{D}|\nabla f|^{2} \rightarrow 0
$$


on the other hand, as $B \geq 1$, the volume of $D$ is uniformly bounded from below, which implies that

$$
\int_{D} f^{2} \geq C>0
$$

We conclude that the Rayleigh quotient of $f$ tends to 0 as $b \rightarrow 0$, which shows the assertion.

Example 16. The following example shows that we need to impose some condition on the outer curve in order to get a positive lower bound as in Theorem 2 .

It is an easy and classical fact that, in order to create a small eigenvalue for the Neumann problem, it is sufficient to deform a domain locally, near a boundary point, as indicated by the mushroom-shaped region shown in the figure below. Up to a gauge transformation, we can suppose that the potential $A$ is locally 0 in a neigborhood of the mushroom, and we have to estimate the first eigenvalue of the Laplacian with Dirichlet boundary condition at the basis of the mushroom (which is a segment of length $\epsilon$ ) and Neumann boundary condition on the remaining part of its boundary, as required by Proposition 12 .

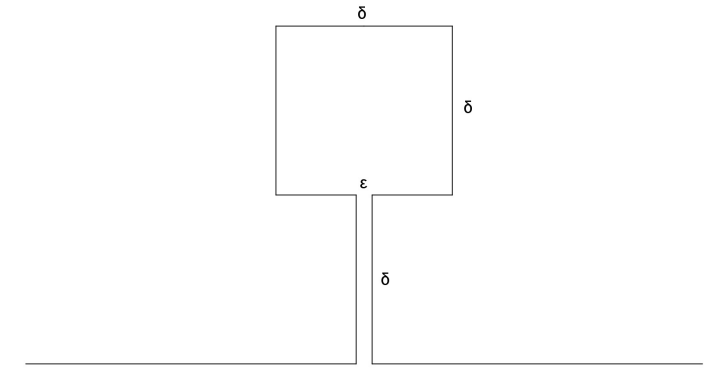

Figure 2: A local deformation implying $\lambda_{1} \rightarrow 0$

The only point is to take the value of the parameter $\epsilon$ much smaller than $\delta$ as $\delta \rightarrow 0$. Take for example $\epsilon=\delta^{4}$ and consider a function $u$ taking value 1 in the square of size $\delta$ and passing linearly from 1 to 0 outside the rectangle of sizes $\epsilon, \delta$. The norm of the gradient of $u$ is 0 on the square of size $\delta$ and $\frac{1}{\delta}$ in the rectangle of size $\delta, \epsilon$.

Then the Rayleigh quotient is

$$
R(u) \leq \frac{\frac{1}{\delta^{2}} \delta \epsilon}{\delta^{2}}=\frac{\epsilon}{\delta^{3}}
$$

which tends to 0 as $\delta \rightarrow 0$.

Moreover, we can make such local deformation keeping the curvature of the boundary uniformly bounded in absolute value (see Example 2 in [6]). 


\section{Appendix}

\subsection{Spectrum of circles and Riemannian products}

We first prove Proposition 7 .

Let then $(M, g)$ be the circle of length $L$ with metric $g=\theta(t)^{2} d t^{2}$, where $t \in[0, L]$ and $\theta(t)$ is periodic of period $L$. Given the 1-form $A=H(t) d t$ we first want to find the harmonic 1-form $\omega$ which is cohomologous to $A$; that is, we look for a smooth function $\phi$ so that $\omega=A+d \phi$ is harmonic. Now a unit tangent vector field to the circle is

$$
e_{1}=\frac{1}{\theta} \frac{d}{d t}
$$

Write $\omega=G(t) d t$. Then

$$
\delta \omega=-\frac{1}{\theta}\left(\frac{G}{\theta}\right)^{\prime}
$$

As any 1-form on the circle is closed, we see that $\omega$ is harmonic iff $G(t)=c \theta(t)$ for a constant $c$. We look for $\phi$ and $c \in \mathbf{R}$ so that

$$
\phi^{\prime}=-H+c \theta \text {. }
$$

As $\phi$ must be periodic of period $L$, we must have $\int_{0}^{L} \phi^{\prime}=0$. As the volume of $M$ is $L$, we also have $\int_{0}^{L} \theta=L$. This forces

$$
c=\frac{1}{L} \int_{0}^{L} H(t) d t .
$$

On the other hand, as the curve $\gamma(t)=t$ parametrizes $M$ with velocity $\frac{d}{d t}$, one sees that the flux of $A$ across $M$ is given by

$$
\Phi^{A}=\frac{1}{2 \pi} \int_{0}^{L} H(t) d t .
$$

Therefore $c=\frac{2 \pi}{L} \Phi^{A}$ and a primitive could be

$$
\phi(t)=-\int_{0}^{t} H+c \int_{0}^{t} \theta .
$$

Conclusion:

- The form $A=H(t) d t$ is cohomologous to the harmonic form $\omega=c \theta d t$ with $c=\frac{2 \pi}{L} \Phi^{A}$.

We first compute the eigenvalues. By gauge invariance, we can use the potential $\omega$. In that case

$$
\Delta_{\omega}=-\nabla_{e_{1}}^{\omega} \nabla_{e_{1}}^{\omega}
$$


Now

$$
\nabla_{e_{1}}^{\omega} u=\frac{u^{\prime}}{\theta}-i c u
$$

hence

$$
\nabla_{e_{1}}^{\omega} \nabla_{e_{1}}^{\omega} u=\frac{1}{\theta}\left(\frac{u^{\prime}}{\theta}-i c u\right)^{\prime}-i c\left(\frac{u^{\prime}}{\theta}-i c u\right) .
$$

After some calculation, the eigenfunction equation $\Delta_{\omega} u=\lambda u$ takes the form:

$$
-u^{\prime \prime}+\frac{\theta^{\prime}}{\theta} u^{\prime}+2 i c \theta u^{\prime}+c^{2} \theta^{2} u=\lambda \theta^{2} u \text {. }
$$

Recall the arc-length function $s(t)=\int_{0}^{t} \theta(\tau) d \tau$. We make the change of variables:

$$
u(t)=v(s(t)), \quad \text { that is } \quad v=u \circ s^{-1} .
$$

Then:

$$
\left\{\begin{array}{l}
u^{\prime}=v^{\prime}(s) \theta \\
u^{\prime \prime}=v^{\prime \prime}(s) \theta^{2}+v^{\prime}(s) \theta^{\prime}
\end{array}\right.
$$

and the equation becomes:

$$
-v^{\prime \prime}+2 i c v^{\prime}+c^{2} v=\lambda v
$$

with solutions :

$$
v_{k}(s)=e^{\frac{2 \pi i k}{L} s}, \quad \lambda=\frac{4 \pi^{2}}{L^{2}}\left(k-\Phi^{A}\right)^{2}, \quad k \in \mathbf{Z} .
$$

Now Gauge invariance says that

$$
\Delta_{A+d \phi}=e^{i \phi} \Delta_{A} e^{-i \phi}
$$

and $v_{k}$ is an eigenfunction of $\Delta_{A+d \phi}$ iff $e^{-i \phi} v_{k}$ is an eigenfunction of $\Delta_{A}$. Hence, the eigenfunctions of $\Delta_{A}$ (where $\left.A=H(t) d t\right)$ are

$$
u_{k}=e^{-i \phi} v_{k}
$$

where $\phi(t)=-\int_{0}^{t} H+c s(t)$ and $c=\frac{2 \pi}{L} \Phi^{A}$. Explicitly:

$$
u_{k}(t)=e^{i \int_{0}^{t} H} e^{\frac{2 \pi i\left(k-\Phi^{A}\right) s(t)}{L}}
$$

as asserted in Proposition 7.

Let us know verify the last statement. If the metric is $g=d t^{2}$ then $\theta(t)=1$ and $s(t)=t$. If $A$ is a harmonic 1-form then it has the expression $A=\frac{2 \pi \Phi^{A}}{L} d t$. Taking into account (19) we indeed verify that $u_{k}(t)=e^{\frac{2 \pi i k}{L} t}$. 
- We now prove Proposition 8 .

Here we assume that $\Omega$ is a Riemannian product $[0, a] \times \mathbf{S}^{1}\left(\frac{L}{2 \pi}\right)$ with coordinates $(r, t)$ and the canonical metric on the circle. We fix a closed potential $A$ on $\Omega$. By gauge invariance we can assume that $A$ is a Coulomb gauge, and by what we said above we have easily

$$
A=\frac{2 \pi \Phi^{A}}{L} d t
$$

Then $A$ restrict to zero on $[0, a]$; as $A(N)=0$ on $\partial \Omega$ the magnetic Neumann conditions reduce simply to $\frac{\partial u}{\partial N}=0$. At this point we apply a standard argument of separation of variables; if $\phi(r)$ is an eigenfunction of the usual Neumann Laplacian on $[0, a]$, and $v(t)$ is an eigenfunction of $\Delta_{A}$ on $\mathbf{S}^{1}\left(\frac{L}{2 \pi}\right)$, we see that the product $u(r, t)=\phi(r) v(t)$ is indeed an eigenfunction of $\Delta_{A}$ on $\Omega$. As the set of eigenfunctions we obtain that way is a complete orthonormal system in $L^{2}(\Omega)$, we see that each eigenvalue of the product is the sum of an eigenvalue in the Neumann spectrum of $[0, a]$ and an eigenvalue in the magnetic spectrum of the circle, as computed before. We omit further details.

\subsection{Proof of Lemma 9}

For simplicity of notation, we give the proof when $a=L=1$. This will not affect generality. Then, assume that $s:[0,1] \times[0,1] \rightarrow \mathbf{R}$ is smooth, non-negative and satisfies

$$
s(0, t)=t, \quad s(r, 0)=0, \quad s(r, 1)=1 \quad \text { and } \quad \frac{\partial s}{\partial t}(r, t) \doteq \theta(r, t)>0 .
$$

Assume the identity

$$
F(t)=p(r) \cos (\pi s(r, t))+q(r) \sin (\pi s(r, t))
$$

for real-valued functions $F(t), p(r), q(r)$, such that $p(r)^{2}+q(r)^{2}>0$. Then we must show:

$$
\frac{\partial s}{\partial r}=0
$$

everywhere.

Differentiate 20 with respect to $t$ and get:

$$
F^{\prime}(t)=-\pi p(r) \theta(r, t) \sin (\pi s)+\pi q(r) \theta(r, t) \cos (\pi s)
$$

and we have the following matrix identity

$$
\left(\begin{array}{cc}
\cos (\pi s) & \sin (\pi s) \\
-\pi \theta \sin (\pi s) & \pi \theta \cos (\pi s)
\end{array}\right)\left(\begin{array}{l}
p \\
q
\end{array}\right)=\left(\begin{array}{c}
F \\
F^{\prime}
\end{array}\right) .
$$


We then see:

$$
p(r)=F(t) \cos (\pi s)-\frac{F^{\prime}(t)}{\pi \theta} \sin (\pi s) .
$$

Set $t=0$ so that $s=0$ and $p(r)=F(0) \doteq p$ is constant; the previous identity becomes

$$
p=F(t) \cos (\pi s)-\frac{F^{\prime}(t)}{\pi \theta} \sin (\pi s) .
$$

Observe that:

$$
\left\{\begin{array}{l}
F^{\prime}(0)=\pi q(r) \theta(r, 0) \\
F^{\prime}(1)=-\pi q(r) \theta(r, 1)
\end{array}\right.
$$

- Assume $F^{\prime}(0)=0$. Then, as $\theta(t, r)$ is positive one must have $q(r)=0$ for all $r$, hence $p \neq 0$ and $F(t)=p \cos (\pi s)$, from which, differentiating with respect to $r$, one gets easily $\frac{\partial s}{\partial r}=0$ and we are finished.

- We now assume that $F^{\prime}(0) \neq 0$ : then we see from (24) that $q$ is not identically zero and the smooth function $F^{\prime}:[0,1] \rightarrow \mathbf{R}$ changes sign. This implies that

- there exists $t_{0} \in(0,1)$ such that $F^{\prime}\left(t_{0}\right)=0$.

Now (23) evaluated at $t=t_{0}$ gives:

$$
p=F\left(t_{0}\right) \cos \left(\pi s\left(r, t_{0}\right)\right)
$$

for all $r$. Differentiate w.r.t. $r$ and get, for all $r \in[0,1]$ :

$$
0=\sin \left(\pi s\left(r, t_{0}\right)\right) \frac{\partial s}{\partial r}\left(r, t_{0}\right)
$$

Since $s(r, t)$ is increasing in $t$, we have

$$
0<s\left(r, t_{0}\right)<s(r, 1)=1 .
$$

Hence $\sin \left(\pi s\left(r, t_{0}\right)\right)>0$ and we get

$$
\frac{\partial s}{\partial r}\left(r, t_{0}\right)=0
$$

(20) writes:

$$
F(t)=p \cos (\pi s)+q(r) \sin (\pi s),
$$

and then, differentiating w.r.t. $r$ :

$$
0=-p \pi \sin (\pi s) \frac{\partial s}{\partial r}+q^{\prime}(r) \sin (\pi s)+\pi q(r) \cos (\pi s) \frac{\partial s}{\partial r} .
$$


Evaluating at $t=t_{0}$ we obtain $0=q^{\prime}(r) \sin \left(\pi s\left(r, t_{0}\right)\right)$ which implies

$$
q^{\prime}(r)=0
$$

hence $q(r)=q$, a constant. We conclude that

$$
F(t)=p \cos (\pi s)+q \sin (\pi s)
$$

for constants $p, q$. We differentiate the above w.r.to $r$ and get:

$$
0=(-\pi p \sin (\pi s)+\pi q \cos (\pi s)) \frac{\partial s}{\partial r}
$$

for all $(r, t) \in[0,1] \times[0,1]$. Now, the expression inside parenthesis is non-zero a.e. on the square. Then one must have $\frac{\partial s}{\partial r}=0$ everywhere and the final assertion follows.

\section{References}

[1] L. Abatangelo, V. Felli, B. Noris, and M. Nys. Sharp boundary behavior of eigenvalues for Aharonov-Bohm operators with varying poles. arXiv:1605.09569, 2016.

[2] W. Ballmann, J. Brüning, and G. Carron. Eigenvalues and holonomy. Int. Math. Res. Not., 12:657-665, 2003.

[3] V. Bonnaillie-Noël, M. Dauge, and N. Popoff. Ground state energy of the magnetic Laplacian on general three-dimensional domains, volume 145. 2016.

[4] V. Bonnaillie-Noël and B. Helffer. Nodal and spectral minimal partitions - The state of the art in 2015. arXiv:1506.07249, 2015.

[5] I. Chavel and E. Feldman. Cylinders on surfaces. Comment. Math. Helv., 53:439-447, 1978.

[6] B. Colbois, A. Girouard, and M. Iversen. Uniform stability of the Dirichlet spectrum for rough outer perturbations. J. Spectr. Theory, 3:575-599, 2013.

[7] M. Egidi, S. Liu, F. Münch, and N. Peyerimhoff. Ricci curvature and eigenvalue estimates for the magnetic Laplacian on manifolds. arXiv:1608.01955, 2016.

[8] L. Erdös. Rayleigh-type isoperimetric inequality with a homogeneous magnetic field. Calc. Var. Partial Differential Equations, 4:283-292, 1996.

[9] B. Helffer, M. Hoffmann-Ostenhof, T. Hoffmann-Ostenhof, and M. P. Owen. Nodal sets for groundstates of Schrödinger operators with zero magnetic field in non-simply connected domains. Comm. Math. Phys., 202:629-649, 1999. 
[10] C. Lange, S. Liu, N. Peyerimhoff, and O. Post. Frustration index and Cheeger inequalities for discrete and continuous magnetic Laplacian. Calc. Var. Partial Differential Equations, 54:41654196, 2015.

[11] R. Laugesen and B.A. Siudeja. Magnetic spectral bounds on starlike plane domains. ESAIM Control Optim. Calc. Var., 21:670689, 2015.

[12] B. Noris, M. Nys, and S. Terracini. On the eigenvalues of Aharonov-Bohm operators with varying poles: pole approaching the boundary of the domain. Comm. Math. Phys., 339:1101-1146., 2015.

[13] I. Shigekawa. Eigenvalue problems for the Schrödinger operator with the magnetic field on a compact Riemannian manifold. J. Funct. Anal., 75:92-127, 1987.

Bruno Colbois

Université de Neuchâtel, Institut de Mathématiques

Rue Emile Argand 11

CH-2000, Neuchâtel, Suisse

bruno.colbois@unine.ch

Alessandro Savo

Dipartimento SBAI, Sezione di Matematica

Sapienza Università di Roma, Via Antonio Scarpa 16

00161 Roma, Italy

alessandro.savo@sbai.uniroma1.it 